\title{
Estudio de la combinación de los procesos de afinamiento de grano de colada y filtrado en latones $^{(\bullet)}$
}

\author{
O. Bustos ${ }^{(*)}$, R. Mannheim ${ }^{(*)}$ y L. Cruz ${ }^{(*)}$
}

\begin{abstract}
Resumen Se consideró la evaluación de diferentes parámetros y propiedades en latones binarios 70/30 y 63/37 previamente caracterizados y sometidos posteriormente a un afinamiento de grano (por medio de adiciones químicas) y/o filtrado. Adicionalmente, se realizaron estudios de microscopía electrónica de barrido (MEB) tendentes a esclarecer el mecanismo de afinamiento de grano, presente en este proceso. Los resultados obtenidos a partir del afinamiento de grano, y especialmente del acoplamiento de los procesos en cuestión, muestran una disminución importante del tamaño de grano y una mejora en las propiedades mecánicas de ambas aleaciones. Debido a la complejidad de las aleaciones base cobre es necesario considerar una serie de factores para esclarecer el mecanismo de afinamiento de grano y filtrado conjunto. El presente trabajo muestra que existe una alta probabilidad de que el zirconio, en presencia de fósforo, magnesio, hierro, azufre, nitrógeno y oxígeno, sea efectivo para producir afinamiento de grano en latones.
\end{abstract}

Palabras clave: Latones. Filtrado. Afinamiento de grano. Propiedades mecánicas. Solidificación

\section{Study of the combination of cast grain refinement and filtered processes on brasses}

\begin{abstract}
This work considered the evaluation of different parameters and properties of binary brasses 70/30 and $63 / 37$. These materials were previously characterized and then submitted to grain refinement (by mean of chemical additions) and/or filtered. Additionally, SEM observations were carried out to identify the grain refinement mechanisms involved in this process. The results obtained from the grain refinement and especially from the combination of both processes show an important grain size decrease and better mechanical properties. Due to the complexity of the $\mathrm{Cu}$-based alloys, it can be concluded that it is necessary to take into account many factors to understand the mechanisms of the grain refinement-filtered process. The present work shows that probably zirconium (in presence of phosphorus, magnesium, iron, sulphur, nitrogen and oxygen) could be an effective grain refiner to produce grain refinement in brasses.
\end{abstract}

Keywords: Brasses. Filtered. Grain refinement. Mechanical properties. Solidification.

\section{INTRODUCCIÓN}

Las importantes ventajas que ofrecen los procesos de filtrado y afinamiento de grano de colada en aleaciones base cobre, han generado, durante los últimos veinte años, gran interés por su desarrollo y aplicación. Estos procesos han demostrado que mediante su aplicación es posible mejorar notable-

$(\bullet$ Trabajo recibido el día 18 de enero de 1999 y aceptado en su forma final el 10 de junio de 1999.

(*) Dpto. de Ingeniería Metalúrgica. Universidad de Santiago de Chile. Casilla de Correos 10233. Santiago (Chile).

E-mail: obustos@lauca.usach.cl mente la calidad de piezas tanto de colada como de productos semiterminados.

El afinamiento de grano de colada, principalmente por medio de adiciones químicas, permite generar una estructura de granos finos, debida a un aumento del número de núcleos disponibles al comienzo de la solidificación que trae como consecuencia una reducción del tamaño de los cristales primarios. Lo anterior conduce a una disminución de las macro y microsegregaciones producto de la reducción del espaciado interdendrítico, lo que reduce la tendencia del material al agrietamiento en caliente. Se ha encontrado además, que es posible mejorar la fluidez de la aleación líquida y la 
resistencia a la corrosión. Por otro lado, las técnicas de filtrado, que se emplean frecuentemente tanto en materiales férreos como en no férreos, con el fin de mejorar la calidad de los productos de colada y semiterminados, logran mejorar las propiedades a través de la eliminación de partículas de impurezas e inclusiones del material.

\subsection{Afinamiento de grano}

Actualmente, el conocimiento práctico que se tiene de los procesos de afinamiento de grano y filtrado en aleaciones base cobre es escaso, particularmente en el caso de los latones.

Específicamente en latones, se ha comprobado que la adición de elementos tales como titanio, manganeso, zirconio, hierro, niobio, vanadio y cobalto provocan un afinamiento de grano (1-7). Con respecto a las distintas investigaciones llevadas a cabo, se ha concluido que existen combinaciones de elementos que resultan positivas y otras negativas; por ejemplo, la combinación de titanio y boro muestra un claro afinamiento de grano, mientras que la combinación zirconio y boro resulta negativa (1, 2, 7 y 8). De acuerdo con Cibula (2) y, posteriormente, con Mannheim (9), la adición únicamente de boro no tiene incidencia sobre el afinamiento de grano de aleaciones base cobre, sin embargo, Weber (10 y 11), logró buenos resultados con la adición de boro en latones. Recientemente, Bustos (12) obtuvo resultados muy positivos en cobre puro, a través de la adición de boro, pero en combinación con carbono. Cibula (2) y Brunhuber (13) sostienen que el afinamiento de grano en aleaciones base cobre se basa en la formación de carburos y nitruros de zirconio. Por otra parte, Weber (14) ha encontrado que es posible lograr un afinamiento de grano en aleaciones $\mathrm{Cu}-\mathrm{Sn}, \mathrm{Cu}-\mathrm{Zn}$ y aleaciones para cojinetes base cobre, por medio de la aleación conjunta de zirconio, magnesio, hierro y fósforo. En general, adiciones entre 400 y 600 ppm de zirconio, 200 ppm de fósforo, 200 ppm de magnesio y 200 ppm de hierro, en latones comerciales 70/30 (latón $\alpha$ ) y latones 63/37 (latón $\alpha+\beta$ ), enfriados en moldes metálicos, permiten obtener un grano equiaxial fino, que fluctúa entre los 150 y $50 \mu \mathrm{m}$; respectivamente (14).

Cabe señalar que, a pesar de los buenos resultados obtenidos a través de la técnica de afinamiento de grano, pueden presentarse algunos inconvenientes. Un ejemplo es el alto nivel de microporosidades concentradas específicamente en bordes de grano, las que asociadas a un exceso de óxidos que generan una aglomeración de partículas facilitan la descohesión de borde de grano.

Por otro lado, el mecanismo de afinamiento de grano en aleaciones base cobre, por medio de nucleantes, no ha sido del todo esclarecido. En el caso de los latones con contenidos de zinc entre 37 y $39 \%$ se estableció (14) que para la transformación $\mathrm{L}+\alpha \rightarrow \beta$, la mojabilidad de la superficie de $\alpha$ debida a la presencia de óxidos y sulfuros $\left(\mathrm{ZrO}_{2}\right.$, $\mathrm{ZrS}_{2}, \mathrm{MgO}, \mathrm{MgS}, \mathrm{P}_{2} \mathrm{O}_{5}$, tensoactivos) induce el efecto nucleante de compuestos intermetálicos, tales como $\mathrm{ZrN}$ y $\mathrm{Fe}_{2} \mathrm{Zr}$, y probablemente $\mathrm{ZrP}$ y $\mathrm{Fe}_{3} \mathrm{P}$, facilitando la nucleación de la fase $\beta$. Podría decirse que $\beta$ actuaría como un inhibidor al crecimiento de $\alpha$, ya que envuelve a esta última. Sin embargo, se demostró que la principal función de los compuestos formados es la de nucleación y no la de inhibición al crecimiento de grano.

Debido a la alta sensibilidad que presentan las aleaciones base cobre al afinamiento de grano, la reproducibilidad de los resultados es compleja; principalmente debido a la acción de las impurezas, algunas de las cuales pueden tener un efecto catalizador y otras un efecto inhibidor al proceso de afinamiento. Por ello, es absolutamente necesario una caracterización química completa previa a cualquier selección de posibles afinantes.

\subsection{Filtrado}

El uso de filtros cerámicos, tanto en aleaciones férreas como no férreas, tiene por objetivo reducir el contenido de inclusiones, lo que se traduce en una serie de ventajas importantes, que en síntesis permiten mejorar la calidad de las piezas fundidas. Entre estas ventajas destacan: el mejoramiento de la terminación superficial, el aumento de la maquinabilidad y de las propiedades mecánicas. En el caso de latones comerciales, se ha demostrado que el uso de filtros de espuma cerámica mejora la fluidez y la resistencia al fisuramiento en caliente. Sahoo et al. (15 y 16), encontraron que con el uso de filtros cerámicos se reducen significativamente las inclusiones de óxidos en aleaciones de cobre, lográndose una disminución importante en la susceptibilidad al fisuramiento en caliente. Los autores no utilizaron elementos afinantes, actuaron sólo sobre la velocidad de enfriamiento. Investigaciones de Simmons et al. (17) muestran que el proceso de filtrado en bronces al aluminio y al manganeso mejora la resistencia a la tracción en un rango entre 30 y $60 \%$. En el campo de la producción de semiterminados de aleaciones base cobre no se ha implementado la técnica de filtrado, a diferencia del aluminio y sus aleaciones, donde las ventajas han sido demostradas ampliamente.

En los trabajos realizados en aleaciones de aluminio (18-21), se ha observado la retención de fundentes y escorificantes además de óxidos propios del proceso de fusión en las cerámicas, que conducen a una mejoría de un 20 a un $50 \%$ en las 
propiedades mecánicas dependiendo del tipo de aleación y de las condiciones de fusión.

Sin embargo, a pesar de las ventajas anteriormente expuestas del proceso de filtrado, su práctica puede presentar algunos problemas tales como la disminución del número de núcleos potenciales y el aumento de la temperatura de colada para filtros de espuma cerámica. Esto último aumenta la solubilidad de gases durante el estado líquido de las aleaciones y genera un aumento de la porosidad de las piezas coladas.

Desde el punto de vista técnico, existe una serie de alternativas en cuanto al método de filtrado y tipo de filtro que pueden ser utilizados. Sin embargo, el método más efectivo en el atrapamiento de impurezas o inclusiones de tamaños superiores a las $30 \mu \mathrm{m}$, es el método de filtración por la formación de una película o cáscara en la superficie del filtro. El filtro más común, asociado a este método, es el de espuma cerámica. De acuerdo a la temperatura de colada, los filtros de espuma cerámica pueden clasificarse en varios tipos diferentes de los cuales los más importantes son: (a) Filtros base $\mathrm{SiC}$, los cuales han sido diseñados para ser utilizados a una temperatura máxima de colada de aproximadamente $1.500{ }^{\circ} \mathrm{C}$, (b) Filtros base $\mathrm{ZrO}_{2}$, los que se recomiendan para temperaturas máximas de colada de $1.600{ }^{\circ} \mathrm{C}$, y (c) Filtros base $\mathrm{MgO}$ y $\mathrm{ZrO}_{2}$ que de entre los mencionados está diseñado para las temperaturas de colada más elevadas, con un máximo de $1.760{ }^{\circ} \mathrm{C}$. En particular, para el aluminio, donde la temperatura de colada no supera $\operatorname{los} 750^{\circ} \mathrm{C}$ (excepcionalmente para ciertas aleaciones se tienen temperaturas de colada de hasta $925^{\circ} \mathrm{C}$ ), se utilizan filtros de espuma cerámica base $\mathrm{Al}_{2} \mathrm{O}_{3}$. En el caso de aleaciones de cobre, específicamente en el caso de los latones y bronces, los filtros más usados son los de $\mathrm{SiC}$.

La porosidad de los filtros es otra variable que debe considerarse en el momento de seleccionar el filtro más adecuado en una aplicación en particular. El tamaño de los poros de los filtros de espuma cerámica varía comúnmente entre 10 y 30 ppi (poros por pulgada lineal), y está directamente relacionado con la efectividad con la que las inclusiones no metálicas puedan ser atrapadas. Según los tamaños anteriormente señalados, estos filtros pueden ser efectivos para partículas de un tamaño promedio de $30 \mu \mathrm{m}$, sin embargo, pueden llegar a atrapar inclusiones tan pequeñas como de 0,5 a $1 \mu \mathrm{m}$.

\subsection{Combinación de los procesos de filtrado y afinamiento de grano}

La idea de aplicar en forma conjunta estos procesos es nueva. Debido a ello, la disponibilidad de antecedentes es prácticamente nula, lo que de alguna forma genera un interés especial desde el punto de vista de la investigación y desarrollo. Sin embargo, sobre la base de los conocimientos teóricos y prácticos que se tiene de los procesos individuales, se espera que los resultados obtenidos en el acoplamiento demuestren las ventajas y potencialidades de esta nueva técnica.

Específicamente se espera que la aplicación conjunta de los procesos de filtrado y afinamiento de grano, en piezas terminadas y productos semiterminados de aleaciones base cobre, permitan:

- Disminución de segregación.

- Disminución de la tendencia al fisuramiento en caliente.

- Aumento de las propiedades mecánicas.

- Disminución en la dispersión de las propiedades del material.

- Mejor respuesta al mecanizado, con un fuerte aumento en la vida útil de la herramienta de corte.

- Aumento de la duración de los rodillos de lami'nación y matrices de extrusión.

- Disminución de las tasas de rechazo.

- Mejoramiento de la terminación superficial y, por consiguiente, mejora en las respuestas a los tratamientos de recubrimiento.

Tal como se planteó anteriormente, no existen antecedentes en la bibliografía sobre la aplicación conjunta de ambos procesos en aleaciones base cobre, sin embargo, es posible esperar un efecto aditivo de las ventajas de ambos procesos individuales basándose en experiencias parecidas obtenidas en la industria del aluminio en la cual se aplican procesos de filtrado posteriores al tratamiento de afino de grano por medio de $\mathrm{TiB}_{2}$. Cuando los procesos se aplican en forma conjunta, las ventajas de cada uno de ellos permiten anular sus aspectos negativos individuales.

\section{PARTE EXPERIMENTAL}

El desarrollo de las experiencias de afinamiento de grano y filtrado, en forma separada y conjunta, se llevó a cabo previa caracterización de las aleaciones en estudio, cuya composición química se resume en la tabla I. Las condiciones de colada, se dan en la tabla II. Por ello, en las experiencias de afinamiento de grano y en acoplamiento de los procesos en cuestión, el tiempo de residencia de los afinantes en el baño metálico fue de $5 \mathrm{~min}$. Cabe señalar, además, que en todas las coladas realizadas, se desgasificó con argón durante $15 \mathrm{~min}$, para evitar la descincificación de las aleaciones.

La adición de los elementos afinantes al baño durante las experiencias correspondientes fue de 
TABLA I.- Composición química de las aleaciones en estudio no tratadas

TABLE I.- Chemical composition of non treated alloys

\begin{tabular}{|c|c|c|c|c|c|c|c|}
\hline \multirow{2}{*}{$\begin{array}{c}\text { Aleación } \\
\text { tipo }\end{array}$} & \multicolumn{6}{|c|}{ Composición química } \\
\cline { 2 - 8 } & \multicolumn{3}{|c|}{ \% en peso } & \multicolumn{4}{c|}{$\mathrm{ppm}$} \\
\cline { 2 - 8 } & $\mathrm{Cu}$ & $\mathrm{Zn}$ & $\mathrm{Sn}$ & $\mathrm{Fe}$ & $\mathrm{P}$ & $\mathrm{Zr}$ & $\mathrm{Mg}$ \\
\hline Latón $70 / 30$ & 70,3 & 29,7 & - & 153 & 35 & 10 & 5 \\
Latón 63/37 & 63,6 & 36,4 & - & 200 & 72 & 10 & 5 \\
\hline
\end{tabular}

TABLA II.- Condiciones de colada de las aleaciones no tratadas

TABLE II.- Cast conditions of non treated alloys

\begin{tabular}{|c|c|c|c|}
\hline \multirow{2}{*}{ Aleación tipo } & \multicolumn{3}{|c|}{ Condiciones de colada } \\
\cline { 2 - 4 } & $\begin{array}{c}\text { Temperatura } \\
\text { líquidus, }{ }^{\circ} \mathrm{C}\end{array}$ & $\begin{array}{c}\text { Sobrecalentamiento } \\
\text { de colada, }{ }^{\circ} \mathrm{C}\end{array}$ & $\begin{array}{c}\text { Temperatura } \\
\text { de colada, }{ }^{\circ} \mathrm{C}\end{array}$ \\
\hline Latón $70 / 30$ & 980 & 100 & 1.080 \\
Latón $63 / 37$ & 900 & 100 & 1.000 \\
\hline
\end{tabular}

600 ppm de Zr, 200 ppm de P y 200 ppm de $\mathrm{Mg}$, a partir de las aleaciones madre $\mathrm{Cu}-33 \% \mathrm{Zr}, \mathrm{Cu}-14$ $\% \mathrm{P}$ y $\mathrm{Cu}-10 \% \mathrm{Mg}$, respectivamente. No se adicionó hierro, ya que éste se encontraba en la aleación como impureza.

El modelo Keel Block, que se muestra en la figura 1, para la obtención de probetas de tracción, utilizado en la mayoría de las experiencias, a excepción del filtrado puro y el acoplamiento de los procesos, donde se introdujeron cambios en el diseño del sistema de alimentación y filtrado, tal como se muestra en la figura, corresponde a una recomendación práctica descrita en la norma ISO R1338 para la obtención de probetas de tracción a partir de aleaciones fundidas base cobre, coladas en moldes de arena (22 y 23). El diseño recomendado consiste en la modificación del modelo original, descrito en la norma ASTM B208 (24), donde además se incluyen las medidas de la probeta estándar para tracción utilizada en este trabajo. Tal como se observa en la figura 1, el filtro se ubicó en forma vertical en el canal de alimentación previo al canal de distribución. El filtro fue precalentado a aproximadamente $300{ }^{\circ} \mathrm{C}$ en un hornillo a gas. La fusión de las aleaciones se llevó a cabo en un horno de inducción de $30 \mathrm{~kg}$ de capacidad. El tamaño de grano se midió por el método del diámetro promedio. Para su determinación sólo se consideraron los granos equiaxiales. Con el fin de minimizar el error se efectuaron 15 medidas a lo largo de los ejes horizontales y verticales de las muestras de sección circular; finalmente, se obtuvo el diámetro promedio de los granos.

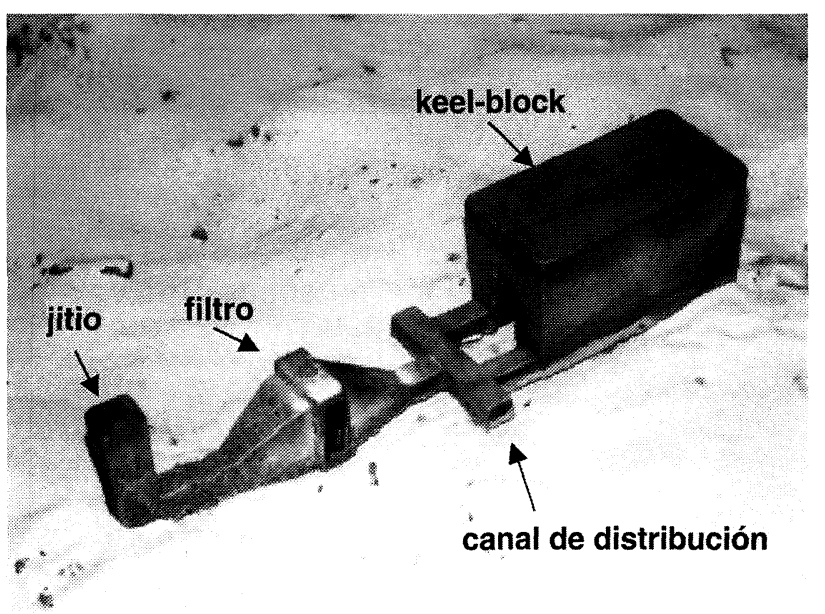

FIG. 1.- Modelo Keel Block modificado con filtro incorporado.

FIG. 1.- Keel Block modified model with incorporated filter.

\section{RESULTADOS Y DISCUSION}

A continuación se muestra el efecto de los procesos de afinamiento de grano y/o filtrado sobre el tamaño de grano, límite elástico, resistencia a la tracción (UTS) y dureza (Figs. 2 a 5). Con relación al tamaño de grano, la figura 2 y las macrofotografías de la figura 3 muestran claramente el efecto de la aplicación de los distintos procesos en forma conjunta.

En el caso del latón 70/30, se observa en la figura 2 una disminución en el tamaño promedio de los 


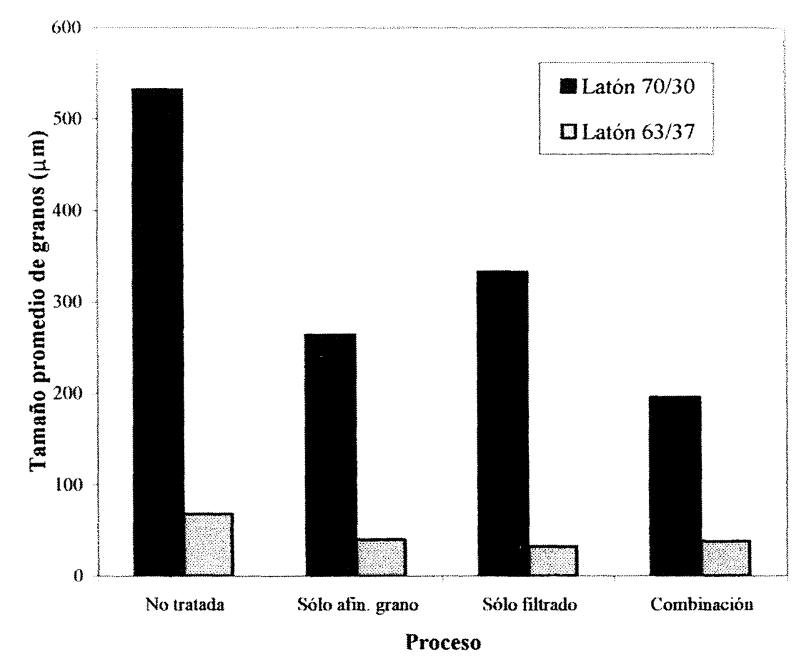

FIG. 2.- Efecto de los procesos de filtrado y afinamiento de grano sobre el tamaño de grano de colada de los latones.

\section{FIG. 2.- Effect of the filtered and grain refinement processes on cast the brasses grain size.}

granos cercano a un $50 \%$ (desde $530 \mu \mathrm{m}$ en la aleación no tratada hasta $270 \mu \mathrm{m}$ para la aleación con tratamiento de afino de grano), lográndose, además, una equiaxialidad (porcentaje de granos equiaxiales) de un $100 \%$ como se aprecia en la figura 3, lo que demuestra la efectividad del proceso. Weber (14)

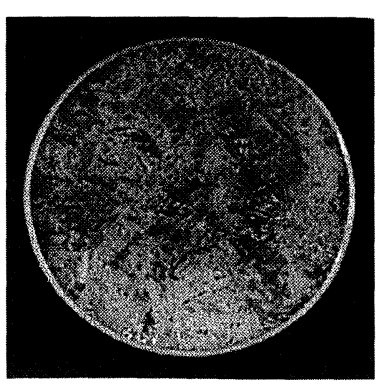

a)

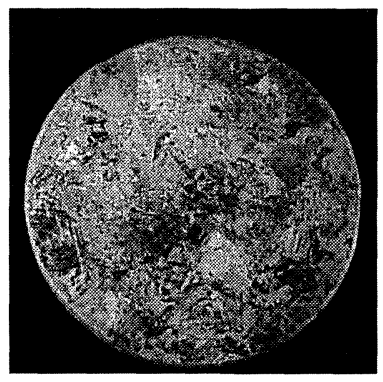

c)

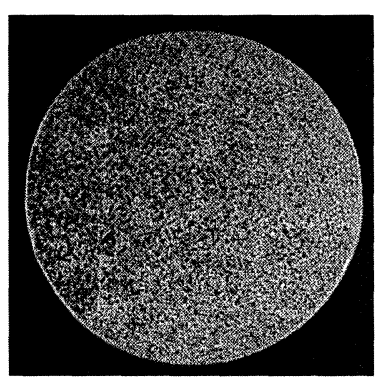

b)

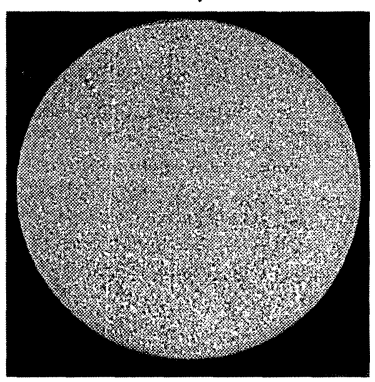

d)
Fig. 3.- Fotomacrografías de un latón 63/37, (a) no tratado, (b) sólo con afinamiento de grano, (c) sólo filtrado y (d) filtrado con afinamiento de grano (combinación).

FIG. 3.- Photomacrostructure of 63/37 brass, (a) non treated, (b) only with grain refinement, (c) only filtered and (d) filtered and grain refinement (combination). demostró la efectividad de las adiciones seleccionadas para este trabajo, en el afinamiento de latones mono y bifásicos; con tal motivo, cabe señalar que la efectividad del zirconio, fósforo, hierro y magnesio, agregados simultáneamente, son función de variables de operación tales como la temperatura de colada, el tiempo de permanencia de los afinantes en el baño previo a la colada, la temperatura de la coquilla, etc. Aun cuando el autor antes citado no definió ecuaciones que permitieran cuantificar el efecto de cada una de estas variables, los resultados encontrados indican que tanto la temperatura de precalentamiento de la coquilla (hasta $500{ }^{\circ} \mathrm{C}$ ), así como el tiempo de permanencia de los afinantes en el baño líquido (hasta $900 \mathrm{~min}$ ) provocan una disminución en el efecto de los nucleantes. Algo similar ocurre con la temperatura de colada (sobrecalentamientos hasta $250^{\circ} \mathrm{C}$ ); esto es, altas temperaturas de colada causan una disminución en el efecto afinante de las adiciones. Todas las experiencias se llevaron a cabo en un horno con atmósfera de argón.

En el presente trabajo se demostró la eficacia de las adiciones en condiciones diferentes, esto es, fusión y colada en atmósfera abierta y desgasificado con argón, bajas velocidades de enfriamiento (moldes de arena) y tiempos de residencia de los afinantes en el baño relativamente cortos. Por otro lado, debido al filtrado, la reducción de tamaño de grano alcanza un $37 \%$, aunque la equiaxialidad es nula. La razón del afinamiento de grano observado en este caso podría deberse a un aumento en la velocidad de enfriamiento durante la solidificación, debida a la presencia del filtro de espuma cerámica. En lo relativo a las propiedades mecánicas, el aumento en el límite elástico es de un 35 y $27 \%$, en probetas con afinamiento de grano y filtrado, respectivamente. El afinamiento de grano logrado justificaría el aumento observado en dicha propiedad, debido a la relación existente entre ambos, la cual puede ser expresada por medio de la ecuación de Hall-Petch (25). Con respecto a la resistencia a la tracción (UTS) se observa un aumento del $14 \%$ en probetas fabricadas con la aplicación conjunta de ambos procesos respecto a aquellas no tratadas. Se observa en la misma figura 4 una leve disminución en la resistencia a la tracción en probetas sólo afinadas de grano con respecto a aquellas no tratadas. A partir de esto último, se podría pensar que esta pequeña disminución en la resistencia máxima a la tracción, debida al afinamiento de grano, viene dada por la presencia de un mayor contenido de impurezas o inclusiones, que actuarían como concentradores de esfuerzos y, eventualmente, como vías de propagación de grietas, una vez alcanzados los niveles de esfuerzo máximos. Por otro lado, la posible presencia de microporos, defecto asociado normalmente a un afinamiento de grano, podría ser un motivo más que justificaría el efecto antes 


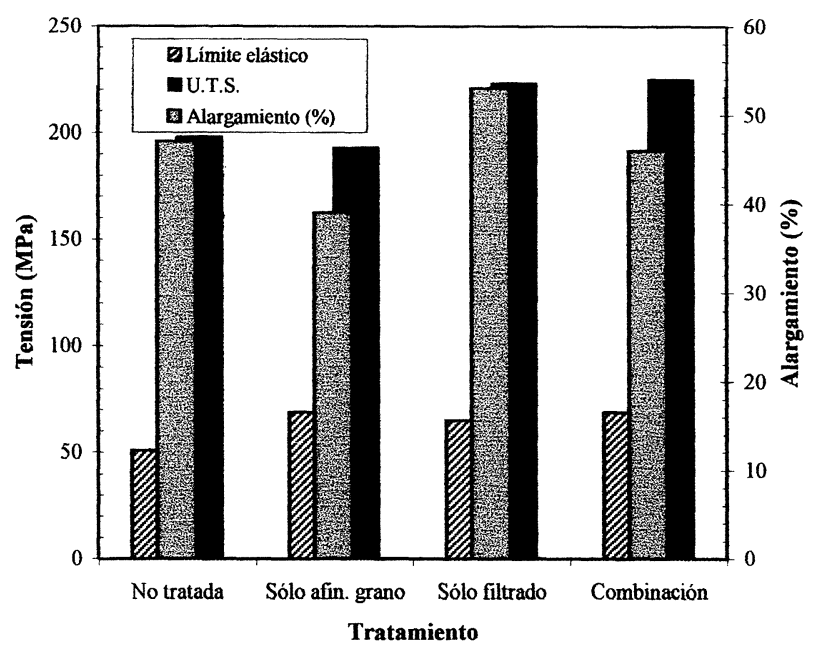

FIG. 4.- Efecto de los procesos de filtrado y afinamiento de grano sobre la resistencia mecánica del latón 70/30.

FIG. 4.- Effect of the filtered and grain refinement processes on the mechanical stregth of $70 / 30$ brass.

mencionado. Este hecho, en parte, pudo ser corroborado por observaciones efectuadas durante el ensayo de tracción, donde se detectó un nivel de agrietamiento superficial bastante inusual. Lo anterior queda reafirmado, además, por los valores de alargamiento mostrados en la figura 4 . Producto de ello, se adoptaron medidas de control más rigurosas, respecto del tiempo de desgasificado, aumentándolo desde 5-10 min hasta 15-20 min aproximadamente. En el caso del filtrado, sin afinamiento de grano previo, el aumento de las propiedades se basa en una mayor limpieza del baño metálico. Esto se observa claramente en los valores correspondientes al alargamiento y a la resistencia a la tracción (UTS) mostrados en la figura 4. La figura 5 muestra

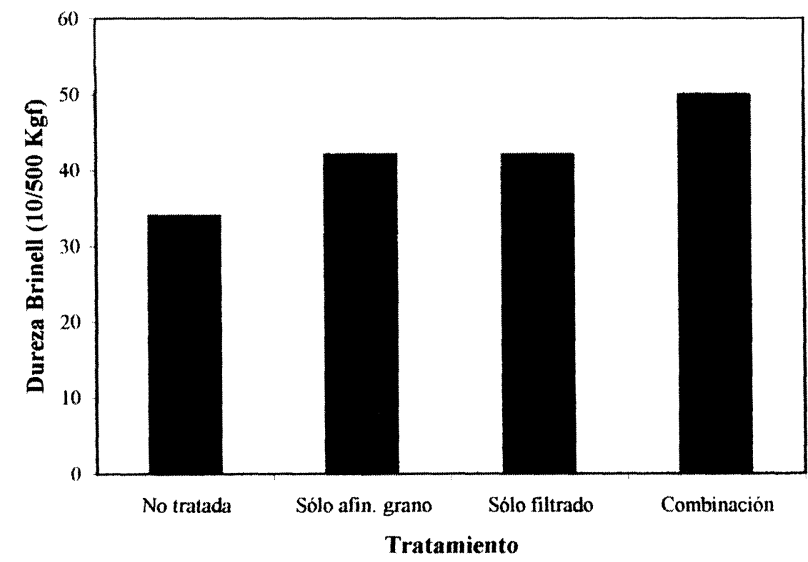

FIG. 5.- Efecto de los procesos de filtrado y afinamiento de grano sobre la dureza del latón 70/30.

FIG. 5.- Effect of the filtered and grain refinement processes on the hardenss of 70/30 brass. el efecto de la aplicación de los procesos separadamente y en forma conjunta sobre la dureza; se observa un aumento significativo de esta propiedad en forma análoga a las otras propiedades mecánicas.

El efecto del acoplamiento de los procesos en el caso del latón 70/30, se resumen gráficamente en la figura 6 . Se muestran los aumentos o disminuciones en porcentaje en las propiedades con relación a las aleaciones no tratadas. El tamaño de grano disminuye en un 50, 37, y $63 \%$ en las aleaciones sólo afinadas, sólo filtradas, y afinadas y filtradas, respectivamente, con respecto a las no tratadas. Por otro lado, el aumento en el límite elástico y en la resistencia máxima a la tracción (UTS), son significativos respecto de las probetas sin tratamiento, lográndose un incremento de hasta el $35 \%$ para el límite elástico. Finalmente, se observa un aumento importante en la dureza, cercano al $50 \%$ con relación al material sin tratamiento, manteniéndose en los otros dos casos un incremento, no menos importante, del $20 \%$.

De acuerdo con lo anterior, se puede afirmar que el efecto del acoplamiento de los procesos de filtrado y afinamiento de grano, en el caso del latón 70/30 es altamente beneficioso.

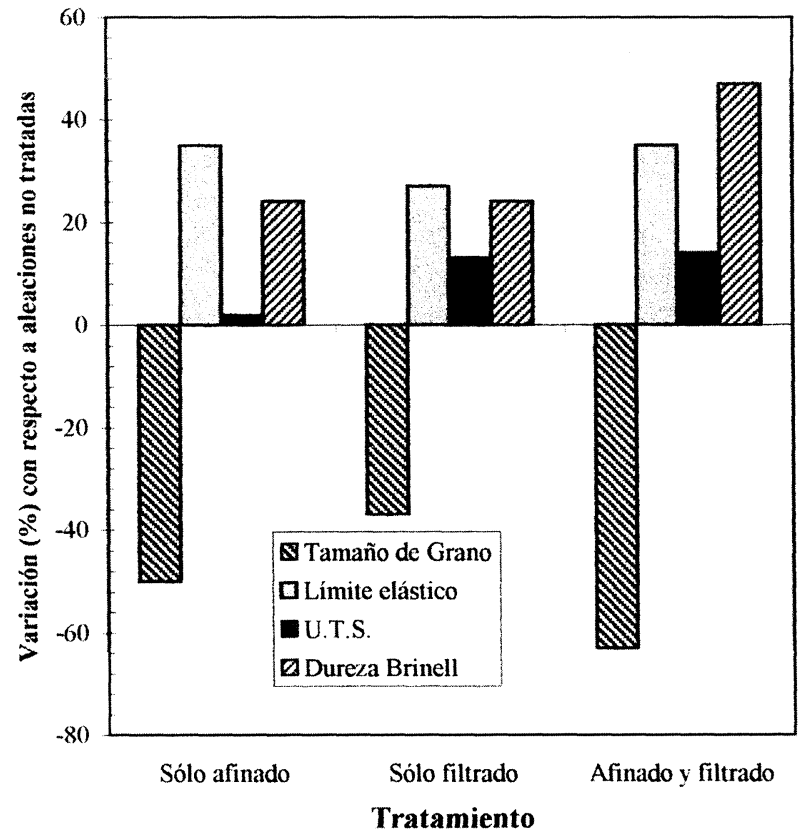

Fig. 6.- Efecto de la combinación de los procesos de filtrado y afinamiento de grano sobre la variación relativa del tamaño de grano y propiedades mecánicas de un latón 70/30.

FIG. 6.- Effect of the combination of filtered and grain refinement processes on the relative variation of grain size and mechanical properties of 70/30 brass. 
En la figura 7 se muestran las micrografías obtenidas por medio de microscopía electrónica de barrido a un posible centro de nucleación. De acuerdo con los resultados obtenidos en el microanálisis, mostrados en la figura 8 (barrido lineal), a la partícula de la figura 7 , es posible inferir que la función de nucleación vendría dada por la presencia de compuestos del tipo $\mathrm{ZrP}_{\mathrm{x}}$, con $\mathrm{x}=0,5-1$, (bajo el supuesto de que la partícula podría ser un compuesto intermetálico binario), cuya efectividad se vería favorecida particularmente por la presencia de una película de sulfuros y/u óxidos que rodearía y mojaría la partícula, favoreciendo de esta manera la nucleación y el crecimien-

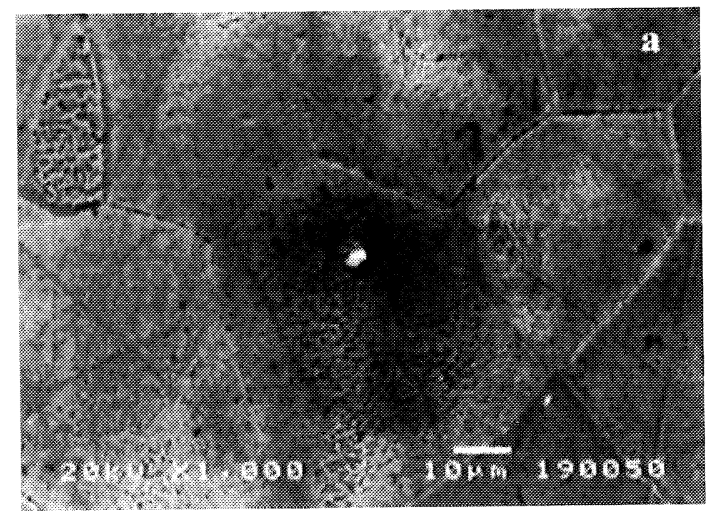

to de la fase sólida. En el caso del latón monofásico, esta fase correspondería a $\alpha$ (fase rica en cobre).

La idea anterior se basa en el hecho conocido de que por medio de un aumento del número de núcleos en el líquido, se puede lograr un afinamiento de grano (1, 2 y 26-29). Ahora bien, el o los mecanismos responsables de este afinamiento de grano, que estarían actuando en forma simultánea, serían la inhibición del crecimiento y la nucleación de los cristales durante la solidificación. En el primero de los casos, el fenómeno de restricción al crecimiento de los cristales, se podría deber a un enriquecimiento de los elementos agregados en el frente de solidificación, lo que provoca un subenfriamiento constitucional, el

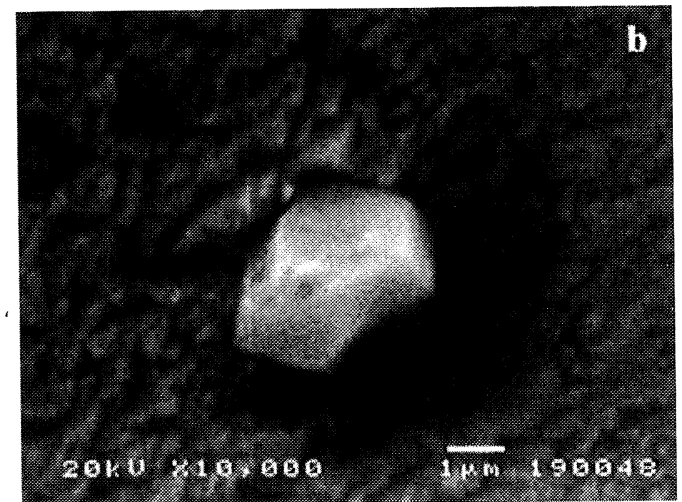

FIG. 7- Micrografías de microscopía electrónica de barrido de un latón 70/30 con afinamiento de grano, (a) presencia de una partícula en el centro de un grano de fase $\alpha$ (matriz) y (b) la misma partícula observada en (a) ampliada 10 veces

FIG. 7.-SEM micrographs of 70/30 brass with grain refinement, (a) particle in the center of a $\alpha$-phase grain (matrix) and (b) same particle $10 \times$ magnified.
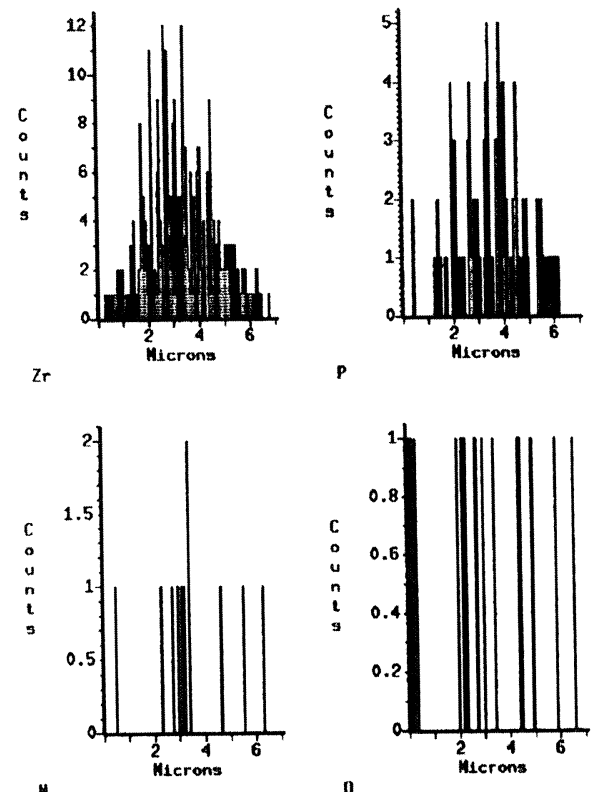

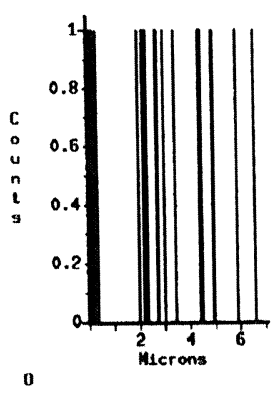

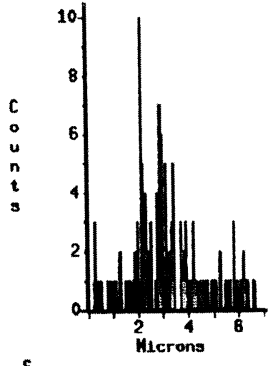

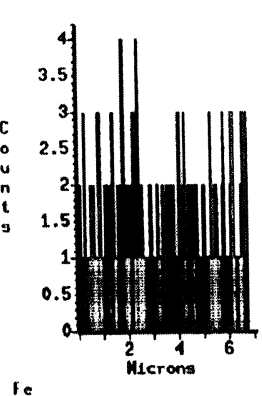

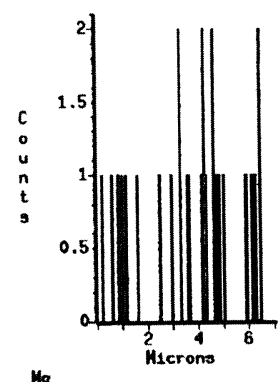

MB

FIG. 8.- Barrido lineal de la partícula observada en la figura 7.

FIG. 8. - Line scan of the particle shown in figure 7. 
cual inhibe el posterior crecimiento de los cristales y posibilita, además, una posterior nucleación del resto del líquido. El segundo mecanismo antes señalado, y que de acuerdo a la literatura (30 y 31$)$ podría corresponder en este caso al compuesto $\mathrm{ZrP}$, supone la presencia de núcleos ajenos (2 y 28), los cuales facilitarían la formación de cristales. Estos núcleos podrían ser partículas de diversa naturaleza, entre las que se pueden encontrar compuestos intermetálicos producto de las adiciones químicas, impurezas existentes en el líquido, o bien partículas ( 2 y 29 ) de cristales formados originalmente y no disueltos completamente. Denison et al. (27) y también Cibula (2), llegaron a la conclusión de que el afinamiento de grano es causado por un lado, a través de una restricción al crecimiento de los granos, y por otro, debido a la presencia de partículas en el líquido, sobre las cuales el líquido puede nuclear.

Los requisitos para que un elemento o compuesto actúe como afinante de grano, han sido presentados en numerosas publicaciones (2, 5, 28 y 32-34). Entre otras, se plantea la necesidad de que existan ciertas similitudes entre los posibles núcleos y el metal que solidifica. Los posibles núcleos deberían tener una idéntica o similar estructura cristalina con el material. Como se mencionó anteriormente, un posible nucleante en latones binarios podría ser el $\mathrm{ZrP}$, cuya estructura cristalina es cúbica de caras centradas, al igual que el cobre, y cuyo parámetro de celda, además, es un $45 \%$ superior que el de éste, factor considerado como negativo en este caso, ya que la diferencia en el parámetro reticular no debería superar el $15 \%$. Sin embargo, este factor por sí solo no es determinante, puesto que según Cibula (2), más importante que el parámetro reticular es la epitaxialidad que se originaría entre los planos densos de la matriz y del nucleante. Estos dos últimos factores permitirían asegurar, en cierta medida, una buena epitaxialidad entre el nucleante y la matriz, es decir, por lo menos algunos planos y direcciones compactas coincidirían entre ambos. Puesto que este requisito no siempre conduce a un afinamiento de grano, no pueden ser excluidos algunos "factores químicos" tales como la concentración de electrones de valencia, efectos de adsorción y las energías superficiales.

En investigaciones previas (2), se ha concluido que debe existir una apreciable cantidad de núcleos para que se lleve a cabo el proceso de cristalización del líquido. A modo de ejemplo, para el caso del TiC en aluminio, Cibula (2) determinó que de 100 núcleos sólo uno era efectivo. De acuerdo con Hencke (5), hay dos posibles causas; la primera, en la que un determinado número de núcleos sea efectivo, en un determinado momento y para un cierto subenfriamiento, en el momento que estos comienzan a crecer se produce un "efecto de escudo o freno" que hace prácticamente imposible una poste- rior nucleación. La segunda causa se relaciona con la nucleación a bajos subenfriamientos, lo que motivaría la inhibición o menor efectividad de otros núcleos que actuarían a mayores subenfriamientos, imposibles de alcanzar.

Debido a la naturaleza compleja de las aleaciones base cobre, se ha llegado a concluir que es necesario considerar una serie de factores para esclarecer el o los mecanismos del afinamiento de grano. En el caso particular de los latones, Weber (14) encontró que no sólo deben considerarse compuestos intermetálicos de carácter simple, sino también óxidos y sulfuros. Cabe señalar que los elementos adicionados presentan una gran afinidad por oxígeno y azufre. Estos resultados han sido corroborados en este trabajo. Las posibles partículas causantes del afinamiento de grano contienen estos elementos como se muestra en las figuras 7 y 8 . El hecho de que el ajuste de celdas cristalinas no deba exceder un $15 \%$, como lo postuló Cibula (2), deja fuera a la mayoría de los posibles compuestos que se formarían en el líquido y que actuarían como núcleos. De acuerdo con los resultados de Weber (14), deben existir entonces otros factores a considerar como se mencionó anteriormente.

En el presente estudio, se ha llegado a la conclusión de que existe una alta probabilidad de que el zirconio, en presencia de fósforo, azufre, oxígeno y magnesio, principalmente, sea un nucleante efectivo para producir un afinamiento de grano óptimo en el caso del latón 70/30.

Para el latón 63/37, se tiene una situación muy similar al caso anterior, aunque la presencia de compuestos tensoactivos, bajo la forma de óxidos y sulfuros, es igualmente probable $\left(\mathrm{ZrO}_{2}, \mathrm{ZrS}_{2}, \mathrm{MgO}\right.$, $\mathrm{MgS}$ y $\mathrm{P}_{2} \mathrm{O}_{5}$ ) tal como lo plantea Weber (14) en su trabajo doctoral.

Tanto en el caso del latón 70/30, como en el latón $63 / 37$, el efecto nucleante de los compuestos intermetálicos está condicionado a determinadas temperaturas de colada y tiempos de residencia para los elementos afinantes (14), por lo que el óptimo en cuanto a tamaño de grano, sólo es válido para una combinación de temperatura-tiempo definida, que para este estudio en particular se detalla en la tabla II.

A partir del análisis de las partículas retenidas en el filtro, en el caso de los latones, se determinó la presencia de los mismos elementos encontrados en los posibles centros de nucleación de análisis anteriores (Figs. 9 y 10). Es probable que estas partículas sean el resultado de la aglomeración de partículas más finas, originadas por un subenfriamiento térmico en el filtro. La naturaleza de estas partículas retenidas confirma el hecho de que los posibles nucleantes están constituidos por los elementos anteriormente señalados. Las mejoras en las propiedades, producto del acoplamiento, se reafirman en el hecho que el filtro retuvo partículas de 

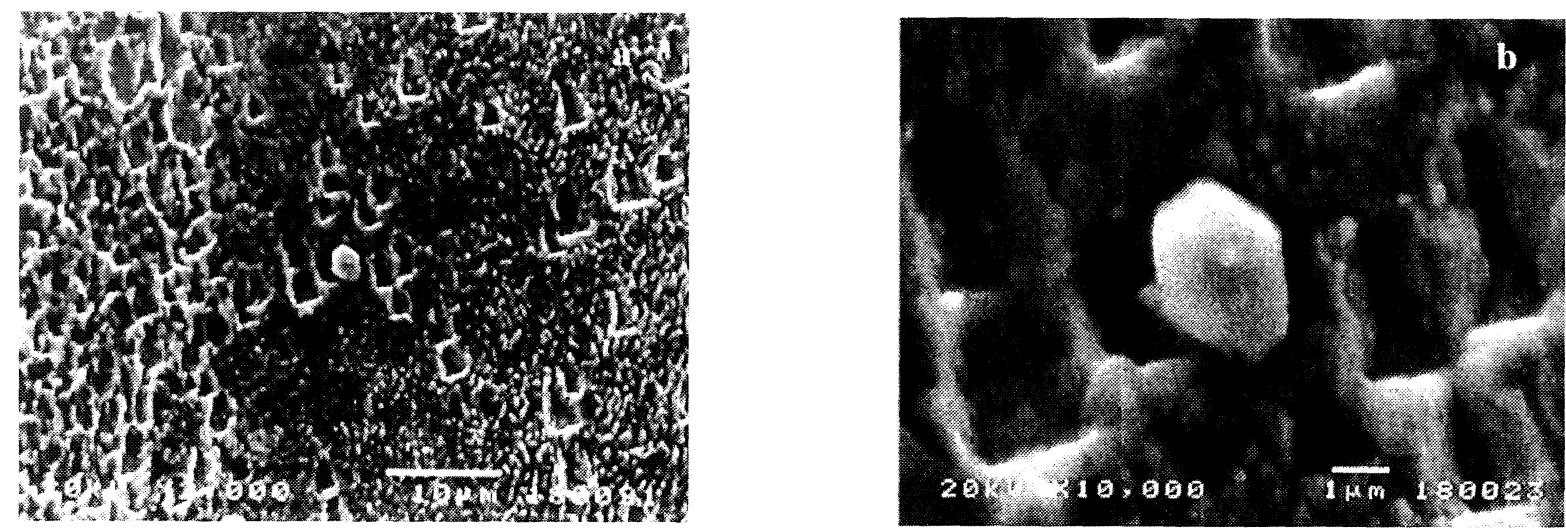

Fig. 9.- Micrografías de microscopía electrónica de barrido de un latón 70/30 con afinamiento de grano, a dos diferentes aumentos correspondientes a la sección tranversal-central de un filtro.

FIG. 9.- SEM micrographs of 70/30 brass with grain refinement, at two different magnifications corresponding to a transversal-central section of a filter.
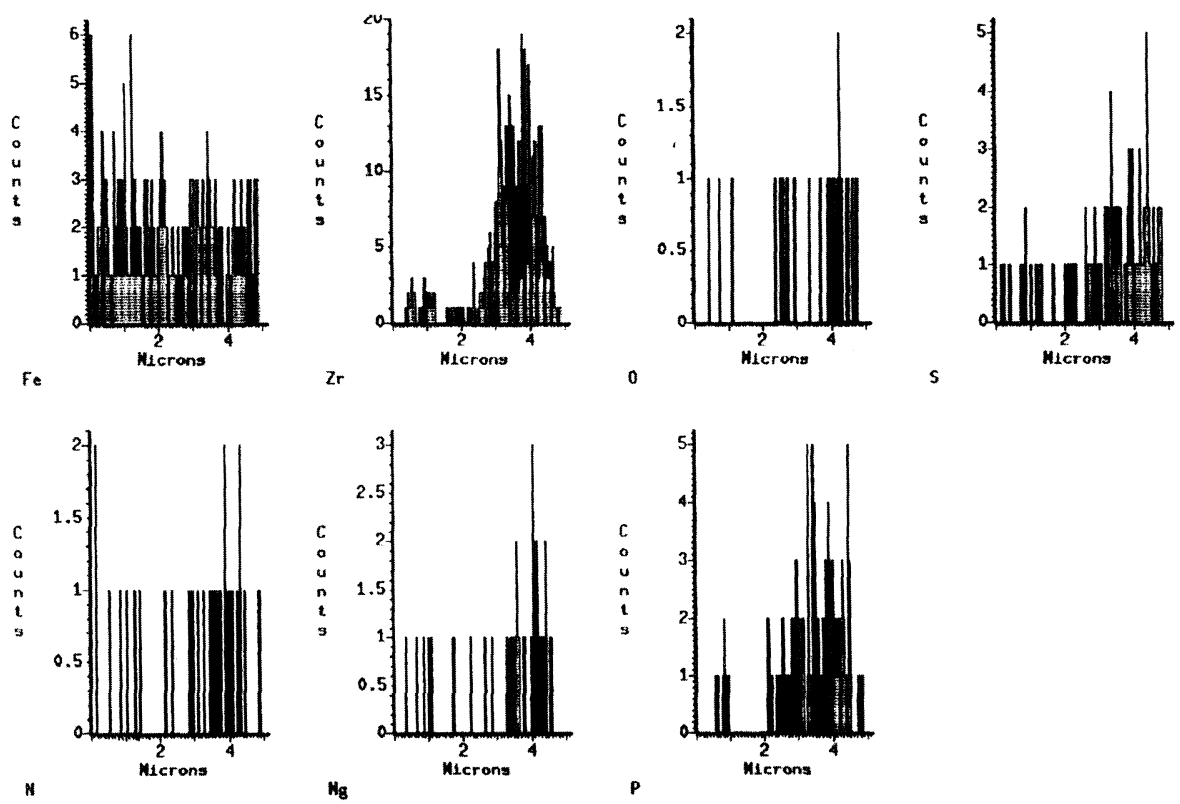

FIG. 10.- Barrido lineal de la partícula observada en la figura 9.

FIG. 10.-Line scan of the particle shown in figure 9.

gran tamaño, que en caso de haber formado parte en el molde habrían provocado un efecto negativo.

En relación con los elementos encontrados, tanto en los probables centros de nucleación como en las partículas retenidas en el filtro, se puede concluir que el zirconio es el elemento principal en el afinamiento de grano de los latones y bronces, como lo confirman investigaciones de diversos autores (1, 2, 5 y 9). Con este motivo, Cibula (2) postula que el ZrC sería el nucleante efectivo, lo cual está en concordancia con lo obtenido por Mannheim (9). En principio, se podría establecer que no sólo carburos sino también nitruros juegan un rol importante. Los resultados del presente trabajo muestran la presencia de un cierto contenido de nitrógeno en los probables centros de nucleación. No se puede, por lo tanto, descartar la formación de $\mathrm{ZrN}$ que también contribuye al afinamiento de grano como lo postuló en su oportunidad Cibula (2). En concordancia con los resultados de Mannheim (9), el fósforo se encontró siempre asociado al zirconio. La presencia de oxígeno y azufre presupone la presencia de óxidos y sulfuros adsorbidos sobre las partículas de fósfuro de circonio ( $\mathrm{ZrP})$. Queda, pues, claramente demostrado en el análisis efectuado por medio de microscopía electrónica de barrido.

Debido a la alta afinidad de las adiciones afinantes con el oxígeno, es de esperar que una fracción 
de éstas reaccione; por ello, no debe descartarse que los productos de estas reacciones también puedan actuar como nucleantes. En este caso, su grado de dispersión y tamaño son de especial importancia. A través de interacciones y aglomeraciones aumentan de tamaño, lo cual queda corroborado en las partículas atrapadas por el filtro cerámico. La convección del líquido debido a la inducción favorece, por otro lado, la aglomeración (35 y 36).

En el presente trabajo, y en contraste con los resultados de Weber (14), el tiempo de residencia fue bastante menor. Weber trabajó en atmósfera controlada (argón) y enfriamientos del material en coquilla: Cortos tiempos de mantenimiento, bajo esas condiciones, no condujeron a un buen afinamiento. Se puede concluir, entonces, que se requiere una cantidad mínima de oxígeno en el baño, que permita alterar la mojabilidad de las partículas afinantes, ya sea adsorbido o formando óxidos. En el presente estudio se trabajó con una atmósfera abierta, por lo cual los tiempos de mantenimiento debieron ser más cortos.

Investigaciones de Joudelis (37 y 38) y de acuerdo a su teoría de la entropía, concluyen que la fuerza motriz de la reacción radica en la diferencia de entropía entre la fase sólida y la fase líquida y, por ello, compuestos con un gran $\Delta \mathrm{S}$ actúan preferentemente en el proceso de nucleación. En vista de la inexistencia de tales datos para estos compuestos complejos de zirconio, magnesio, fósforo, azufre, oxígeno, nitrógeno, y/o carbono, no se pueden efectuar conclusiones definitivas. Cabe señalar, finalmente, que el fundidor tiene sólo un limitado rango de temperatura para alcanzar una estructura de granos finos, sin la adición de nucleantes, lo que, además, depende fuertemente de la velocidad de enfriamiento.

Las adiciones afinantes permiten cubrir un espectro mayor de posibilidades, más aún, si los aspectos negativos de las adiciones, como por ejemplo su aglomeración y generación de inclusiones dañinas, pueden ser eliminadas a través del filtrado, como se demostró en este trabajo.

\section{CONCLUSIONES}

El efecto de los procesos individuales de filtrado y afinamiento de grano, sobre el tamaño de grano y las propiedades mecánicas de los latones ensayados, resultó altamente positivo, especialmente en el caso del latón 70/30.

El efecto de acoplamiento de los procesos de filtrado y afinamiento de grano, sobre el tamaño de grano y las propiedades mecánicas, resultó positivo, también en forma notoria, en el caso de los latones 63/37.

En relación con el mecanismo de afinamiento de grano presente en los latones estudiados, se ha llegado a la conclusión de que existe una alta probabilidad de que el zirconio, en presencia de fósforo, azufre, oxígeno y magnesio, principalmente, sea un nucleante efectivo para producir un afinamiento de grano óptimo.

Las mejoras en las propiedades, producto de la combinación de ambos procesos, se reafirman en el hecho de que el filtro retuvo partículas de gran tamaño, que en el caso de haber ingresado al molde habrían provocado un efecto negativo.

\section{Agradecimientos}

Los autores agradecen al Fondo Nacional de Investigaciones Científicas y Tecnológicas (FONDECYT), a través del proyecto $\mathrm{N}^{\circ} 1961294$ y a la Dirección de Investigaciones Científicas y Tecnológicas de la Universidad de Santiago de Chile, el apoyo económico brindado para su ejecución.

\section{REFERENCIAS}

(1) Henke, R. Giesserei-Prax., 14 (1), 1964: 14-20.

(2) Cibula, A. J. Inst. Met., 82, 1953/54: 513-524.

(3) DiEs, K. Kupfer und Kupferlegierungen in der Technik, Springer Verlag, Berlin-Heidelberg-New York, 1967, 2596.

(4) Schulenberg, A. Schmeltz-und Legierungspraxis in der Metallgiesserei, Fachverlag Schiele und Schon $\mathrm{GmbH}$, 1982: 25-60.

(5) HENKE, R. Giesserei-Prax., (24), 1964: 475-483.

(6) De Galiza, J.A. y Cupini, H.L. Extract No 525067, 9-103.

(7) Couture, A. Giesserei-Prax., (22), 1997: 341-346.

(8) Couture, A. y Edwards, J.O. Giesserei-Prax., (21), 1974: 425-435.

(9) Mannheim, R. y Reif, W. The Solidification of Molten Metals (Proc. Conf.), DGM, (Alemania), 1982: 109-140.2

(10) WeBer, H.G. Giesserei, 69 (3), 1982: 68-72.

(11) WeBER, H.G. Tech. Rundsch., (1/2), 1985: 19.

(12) Bustos, O. Untersuchung zur Kornfeinung von ReinstKupfer durch chemische Zusatze und Deutung der vorgange”. Dr.-Ing. Dissertation, Tesis Doctoral, Technische Universität, Berlín 1990.

(13) Brunhuber, E. Giesserei Prax., (2), 1966, 1721.

(14) Weber, G. Entwicklung eines Kornfeinungszusatzes und Deutung der Vorgange bei der kornfeinung der KupferZinc 30- und Kupfer-Zinc 36- legierungen, Dr.-Ing. Dissertation, Tesis Doctoral, Technische Universität, Berlin 1989: 97-107.

(15) SAHOO, M., BARRY, J.R. y KleinSCMIDT, K. AFS Trans. 1981: 611-620.

(16) LiU, Smith, R.W y SAHoo, 97th Casting Congress and Castexpo ‘93, AFS, Abril 1993.

(17) Simmons, W y Hack, J. Br. Foundryman., mayo 1993.

(18) Ali, S., Apelian, D. y Mutharasan, R. Can. Metall. Q., 24 (4), 1985: 311-318.

(19) Suart, U. Foundry Alloys-Parts I, Foundry $M \& T$, marzo 1985: 25-29. 
(20) Grauchler, L.J., Waeber, M.M., Conti, C. y JacobDuliere M. J. Met., septiembre 1985, 47-50:

(21) NaRdecchia, F. y Mannheim, R. Congreso Internacional de Metalurgia, Lima (Perú), 1988: 1-30.

(22) Couture, A. y Edwards, J.O. AFS Trans., 85, 1977: 97.

(23) Whiting, SAhoo, M. y Brown, D.A. AFS Trans., 85, 1977: 97.

(24) ASTM. B 208-58: Recommended Practice for Tension Test Specimens for Cooper Base Alloys for Sand Casting": 376-381.

(25) Campbell, J. "Casting" 1995.

(26) Wallace, J.F. Vortrag aut dem Internationalen Giessereikongress, Zurich, 1961.

(27) Dennison, J.P. y Tull, E.V., J. Inst. Met., 81, 1952/53: 513.
(28) Rohrig, K. Metall, 20dez 1966: 1266.

(29) Tamman, G. Z. Metallkd., 21 (9), 1929: 277.

(30) International Centre for Diffraction Data. Powder diffraction file, Sets 1-44. 1994.

(31) Shunk, F.A. Constitution of Binary Alloys. Materials Science and Engineering Series. 1969.

(32) SCHEIL, E. Giesserei, 5, 1951, 01.

(33) Turnbull, D. y Vonnegut, B. Ind. Eng. Chem., 44 (6), 1981: 1292.

(34) Tiller, W.A. y Takahashi, T. Acta Met., 17 (8), 1969: 483.

(35) KING, H. Z. Metallkd., 65 (5), 1974: 337.

(36) LANGBein, D. Metall, 38 (5), 1984: 339.

(37) JoudELIS, W.V. Met. Sci., sept. 1979: 540.

(38) JoudELIS, W.V. Aluminium, 57, 1981: 159. 